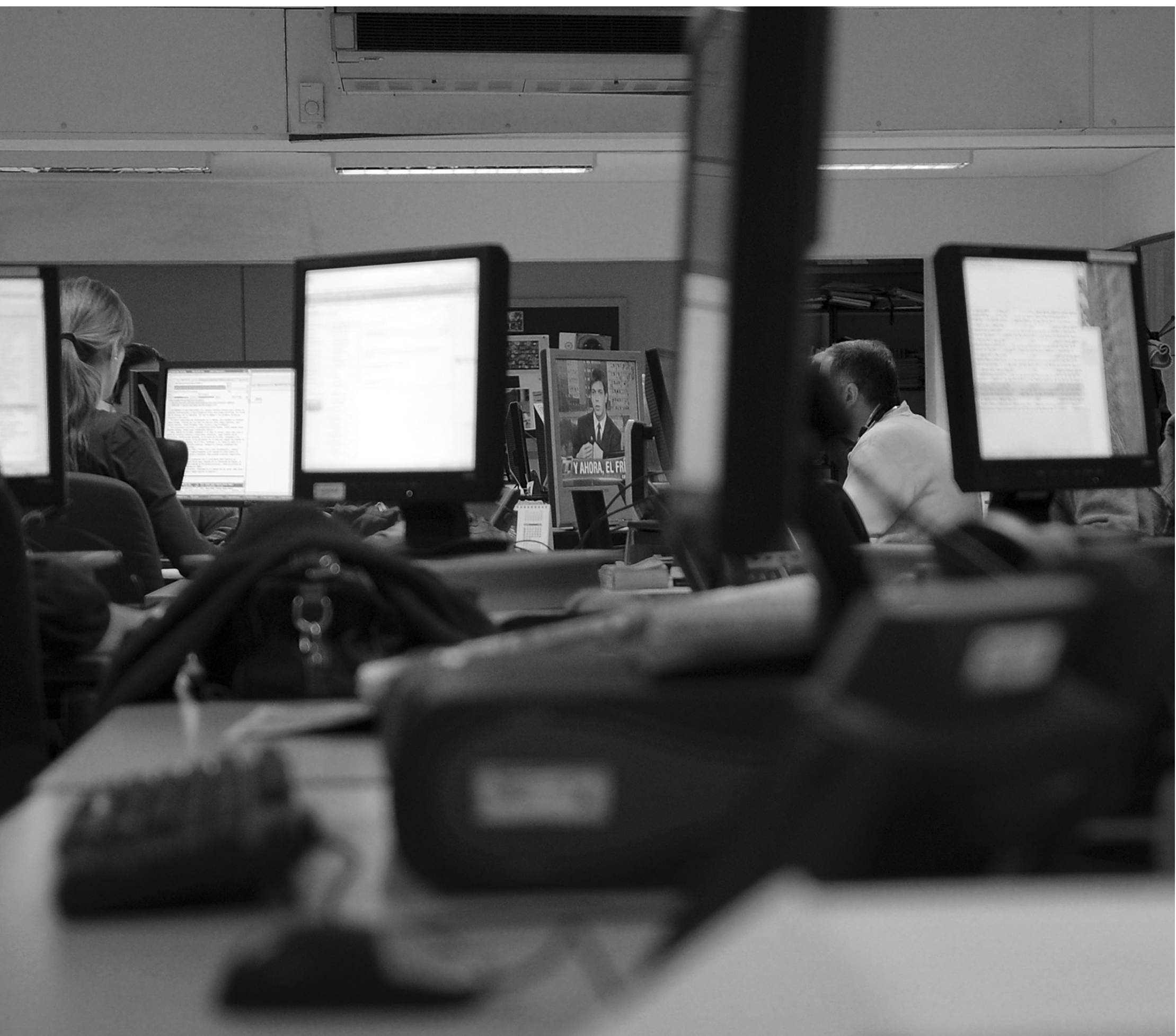




\title{
El sueño de tener una Intranet que funcione como Wikipedia*
}

\author{
Por Diana Blanco, Dafne Lavore \\ y Ana Inés Pepe \\ Fotos de Pablo Porciúncula
}

¿Cuándo Intranet se convierte en un éxito como herramienta de comunicación interna en las organizaciones? ¿Por qué algunas veces es un fracaso? En este artículo, las autoras discuten cómo la transmisión de información trasciende las posibilidades tecnológicas y la voluntad de los directores y depende, también, de las redes de comunicación informal.

¿Por qué poner el foco en las Intranets, las redes informáticas descentralizadas para los empleados de una organización? En nuestra práctica profesional, como comunicadoras organizacionales, nos encontramos con el desafío de implementar, mejorar o asesorar sobre la implementación de una Intranet, y escuchamos, a menudo, de colegas y directivos de empresas la descorazonadora pregunta: “¿Qué falla en nuestra Intranet?” ¿Por

* Wikipedia es una enciclopedia electrónica de contenido libre, que los usuarios pueden editar. http://www.wikipedia.org/ 
qué, a pesar de los grandes esfuerzos que realizan los administradores de contenidos, los integrantes de la organización no se informan a través de ella y, mucho menos, participan en la construcción de los mismos?

Para responder estas y otras preguntas, proponemos un recorrido por las principales conclusiones de investigaciones recientes sobre los usos de Intranet, surgidas de casos concretos, con la intención de descubrir cómo la Intranet impacta en la generación, distribución y aplicación del conocimiento organizacional y por qué en algunos casos esa implementación es un éxito, mientras que en otras es un fracaso.

¿Pueden las organizaciones no tener Intranet? "No podés no estar en Internet", es ya una frase hecha. Y, si bien esto todavía no funciona de la misma manera para Intranet, ${ }^{1}$ lo cierto es que se ha impuesto como una de las manifestaciones más notorias de las nuevas prácticas organizacionales, en el marco de la economía del conocimiento. $^{2}$

La Intranet se entiende como una Internet delimitada solo para los empleados de la organización en la cual se implementa. Las primeras Intranets corporativas empezaron a aparecer hacia principios de 1990 en universidades y empresas de tecnologías, y después se fueron popularizando hacia las grandes empresas. ${ }^{3}$ A través de esta plataforma se pueden difundir las novedades de la empresa (cambios, lanzamientos, noticias sobre productos y servicios, procesos, integrantes), publicar todo tipo de documentos (misión y visión, políticas, manuales, formularios), actualizar constantemente diversos datos (indicadores de ventas, performance, salud laboral), tener un espacio de FAQ, ${ }^{4}$ realizar encuestas, tener un motor de búsqueda, lista de tareas y calendario de eventos. Con la llamada tecnología 2.0, Intranet se está transformando, también, en una herramienta que convierte a los usuarios lectores en usuarios productores y editores de contenido, ${ }^{5}$ además de permitirles personalizar sus interfases, configurar sus propios menús de inicio, participar y administrar grupos, entre otras nuevas aplicaciones.

En un momento histórico en el que Peter Drucker afirma que "lo que hace diferente a la sociedad actual no es que el conocimiento sea otro recurso como la tierra o el capital, sino que es el recurso" y en el que Alvin Toffler dice que "la creación de riqueza depende cada vez más del intercambio de datos, información y conocimiento" ${ }^{\circ}$ (por citar solamente a dos de los autores más difundidos entre quienes han reflexionado sobre la nueva economía), las organizaciones se encuentran con que sus ventajas competitivas estarán basadas en la generación y transferencia de conocimiento, hacia fuera y hacia adentro.

En este escenario, la Intranet 2.0 aparece como la posibilidad de realizar un ideal: la organización en red donde todos los integrantes están conectados, generando y transmitiendo conocimiento. Una organización donde todos acceden a aquella información que antes quedaba encapsulada en cada departamento. Una organización donde se derriban los niveles jerárquicos, con el libre acceso para todos, y donde el organigrama se supera al estar todos en comunicación con todos y que, por lo mismo, generan esa especie de Wikipedia interna a la que, voluntaria y generosamente, todos los integrantes aportan, enriquecen y de la cual se nutren... Pero, lamentablemente, la distancia entre la promesa y la realidad es muy grande.

En muchos de los casos, al breve tiempo de su implementación en la empresa, la Intranet desfallece de soledad y abandono. Sí, ella, que se perfila como el medio de las organizaciones de la nueva economía, dejando en el olvido a la revista interna, y ni que hablar de circulares, memos y afınes, a la que los responsables de su administración y diseño dedican muchas horas y grandes 
esfuerzos, a la que constantemente se le agregan nuevas aplicaciones tecnológicas, a la que se destinan grandes inversiones...

Factores de éxito y de fracaso en Intranet ¿En qué fracasan y qué tanto fracasan las Intranets? Las investigaciones académicas han revelado que el propósito de la implementación de Intranets es mayoritariamente para aumentar la diseminación de información y la integración, para mejorar la comunicación horizontal/vertical y la colaboración, el aprendizaje organizacional y facilitar el gerenciamiento del conocimiento (autores como Telleen; Curry and Stanchich; Ruppel and Harrington; Stenmark; Heide Duane y Finnegan; Damsgaard y Scheepers; Swan). ${ }^{7}$ El conjunto de investigadores que han documentado casos de Intranet dejan constancia que se han conocido muy pocos casos que reporten resultados positivos a partir de los objetivos establecidos y que los fracasos se dan a nivel de la implementación, el desarrollo y la aceptación. ${ }^{8}$

Las investigaciones realizadas, académicas y no académicas, plantean que, más allá de los contenidos y de la tecnología, las Intranets fracasan en el intento de ofrecer un conocimiento accesible y aplicable al usuario. El conocimiento está, el contenido está, pero los integrantes de la organización no llegan a él por varias razones, como la cultura organizacional (cómo se concibe el conocimiento, el empowerment, el control), la apertura comunicacional (la confianza y los flujos entre las comunidades de práctica), las características propias de la Intranet (la usabilidad del diseño y la arquitectura de contenidos). ${ }^{9}$

Hay algunos descubrimientos interesantes sobre los casos de éxito más recientemente investigados, como por ejemplo el tiempo en que una Intranet tarda en convertirse en un caso de éxito: entre 3 y 5 años. Varias investigaciones no académicas, como el Informe de Norman Nielsen ${ }^{10}$ sobre 14 compañias en 6 países, ${ }^{11}$ plantean que una Intranet es un caso de éxito cuando cumple dos metas: 1) que los empleados la usen, es decir, que se informen, se comuniquen, colaboren con otros a través de ella y participen en la generación de sus contenidos; y 2) que su implementación haya contribuido a la mejora de indicadores del negocio. Este último es uno de los aspectos más difíciles de evaluar, aunque sí parece fácilmente verificable el ahorro significativo que se produce al canalizar la documentación de la empresa a través de Intranet (con manuales, formularios, etc.), ya que bajan sensiblemente los costos de impresión y aumenta la productividad (ya no hay que trasladarse, fotocopiar, esperar, etc.). ${ }^{12}$

Algunos autores han discutido la dificultad para medir el impacto económico de la implementación de una Intranet dentro de una empresa. Algunos consideran que proviene de que "las actuales aplicaciones contables que derivan de una etapa económica industrial (caracterizada por el pleno predominio del capital y los activos tangibles como recursos fundamentales), muy diferente a la actual economía de servicios y conocimiento, no son válidas para reflejar el valor real generado por los activos intangibles (...) De hecho, la contabilidad tiene entre sus criterios fundamentales la depreciación y amortización, que dan por supuesto la pérdida progresiva en el tiempo del valor de una inversión por su uso y obsolescencia. Algo contrario a lo que sucede con una Intranet que, por sus características propias, lo normal es que su valor aumente cuanto mayor sea su uso y contenidos". ${ }^{13}$

Para Nielsen, el foco debe estar puesto en la usabilidad, y si analizamos sus conclusiones vemos que el gran valor de una Intranet aparece cuando la gente encuentra en ella el contenido que está buscando y porque puede contribuir a la producción de contenido. Y ese encontrar y generar no responde a las dinámicas de la organización tradicional, industrial, de la economía de Marshall. No responde a la lógica del organigrama, ni del control jerárquico:

"Cuando las arquitecturas de información de la Intranet (...) están estructuradas de acuerdo al diagrama de la organización, los empleados en-
7:: Banck, B. y Amcoff Nyström Ch. 2005. "A study of five Swedish organizations". Journal of Organizational Journal of Organizational Transformation and Socia Change. Vol. 2 No. 2. 8:: lbid. 9:: Ibid 10: Nielsen es el principal directivo del Grupo Nielsen Norman, del cual es cofundador junto al Dr. Donald A. Norman (anteriormente VP de investigación en Apple Computer). Fundo Apple Computer). Fund un movimiento para la implementación rápida y barata de mejorias en las interfaces de usuario y ha inventado varios métodos de usabilidad, incluida la evaluación heurística. 11:: Informe de Nielsen sobre 14 compañias en 6 países: "Social Networking on Intranets". Jakob Nielsen's Alertbox, August 3, 2009. http://www. useit. com/alertbox/social-intranetfeatures.html features. McGovern, G. 2002. "Intranet Return On Investment, case studies". Disponible en: http:// www.gerrymcgovern.com/ nt/2002/nt_2002_11_18 intranet_roi.htm 13: Pérez González, D. y Solana González, P. 2006. "Intranets: medición valoración de sus beneficios en las organizaciones". En: El profesional de la información, septiembre-octubre, Vol. 15, No. 5 , pp. 331-341. 
cuentran muchas dificultades para orientarse. Es mejor estructurar la información de acuerdo a la manera en que la gente hace uso de ella, y no de acuerdo al departamento que la posee (...) Por lo general, los directores principales no están abiertos a las posibilidades de innovación para la empresa 2.0 pues no son usuarios activos de esas herramientas fuera del trabajo. De hecho, muchos altos directivos aún consideran esas herramientas como un juego de niños (...) Cuando se les deja a su propio rumbo, las comunidades se regulan ellas mismas y tienen muy poca necesidad de un estricto control organizacional. Regulaciones peer-to-peer son generalmente más efectivas que un acercamiento a lo Big Brother. Quizás más que cualquier otra innovación corporativa en las Intranets, las tecnologías de software social, están sacando a la luz las carencias de la comunicación corporativa y la colaboración -y por momentos, supliéndolas antes que la compañía (lenta por lo general) pueda comprender (y controlar) completamente el flujo (...) El uso de tecnologías Web 2.0 para comunicarse con los clientes, ha enseñado a muchas compañías que no pueden seguir controlando sus mensajes. Esto se torna más real cuando se analiza el uso de la Web 2.0 para la comunicación interna. Las compañías que una vez sostuvieron el paradigma de mando-y-control para la mensajería corporativa, están viendo cómo cada vez es más difícil mantener esa posición”. ${ }^{14}$

El cambio de las reglas de juego en la sociedad de la información sacude el tablero de juego y hace caer las piezas y procesos que tradicionalmente los vinculaban. La Intranet de una organización que vive en la nueva economía en el 2010 no puede funcionar meramente como una revista online para que la gente lea las novedades de la empresa alimentado por un grupo selecto, validado por las autoridades, sino que debe operar como el sistema nervioso de la organización, en el que, en la versión ideal a la que estos casos de éxito supuestamente se acercan, todos encuentran lo que necesitan, todos se vinculan con todos, todos producen, todos aportan, todos colaboran, sin que medie el control o la censura.
¿Pueden realmente todas las organizaciones llegar a esta Intranet modelo? Como coinciden todas las fuentes (aunque las académicas no tanto) esta pregunta trasciende la tecnología, la cuál por supuesto es una de las variables del problema, pero no la que define lo que estamos denominando "éxito" de una implementación de Intranet. Esa Intranet ideal podrá funcionar en tanto la cultura de la empresa lo habilite, en tanto las estructuras tradicionales se vuelvan orgánicas y permeables y en tanto las características de las tareas y de los integrantes de la organización lo hagan viable. Como dice Nielsen. "La comunicación corporativa debe adaptarse a la cultura en tiempo real de los medios sociales y debe ser mucho más proactiva que en el pasado. Los procedimientos que requerían días o semanas para su aprobación necesitan optimizarse o el tiempo les pasará factura. Nuevamente: el cambio es organizacional y de negocios, no solamente de las herramientas 2.0 por sí solas (...) Si la gente está fuertemente comprometida con el criterio de que 'el conocimiento es poder' y no quiere compartir, entonces las tecnologías del intercambio fallarán irremediablemente". ${ }^{15}$

Intranet en la economía del conocimiento Si partimos de la base de que el conocimiento es el recurso básico de la economía actual y establecemos que una tecnología como Intranet se configura como la herramienta "ideal" para lograr que ese conocimiento se genere y viaje en la organización, entonces ¿por qué un significativo número de los casos documentados coinciden en la dificultad de establecer su éxito y medir su impacto económico?

Un concepto con poder explicativo es el de la paradoja de la productividad, acuñado por Brown y Duguid: "Una clave de la "paradoja de la productividad' de hoy en día (inversiones enormes en nuevas tecnologías, que no muestran grandes crecimientos en la economía) podría ser que la sociedad está todavía luchando para desarrollar instituciones adecuadas a la nueva economía. 


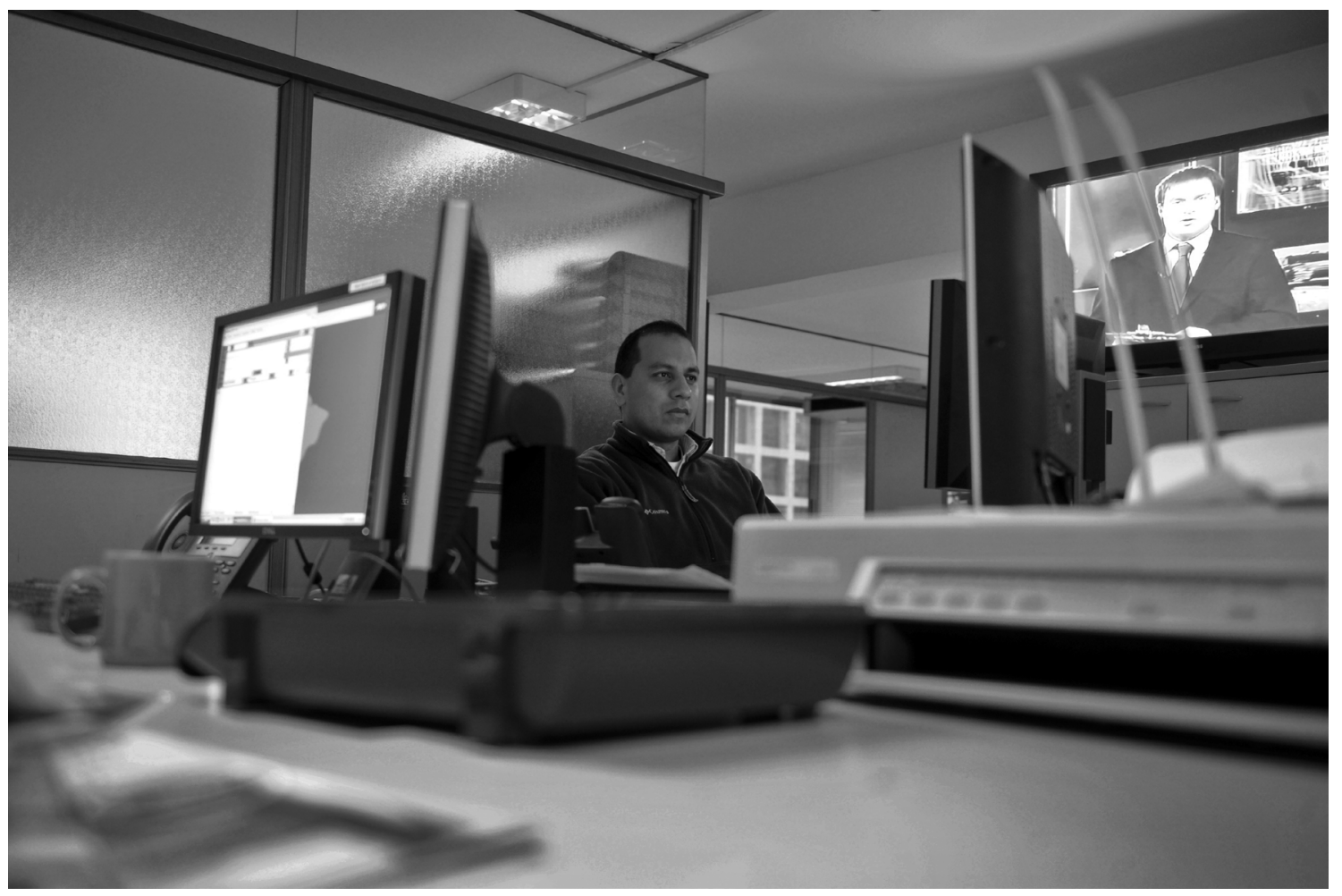

Todo esto podría mostrar una relación compleja entre organizaciones y tecnología, en donde la cruda yuxtaposición de nuevas tecnologías y viejas instituciones es una sobresimplificación del fenómeno". ${ }^{16}$

Como han señalado las investigaciones citadas, Intranet no funciona en una organización que no esté ya configurada para la nueva economía, en una organización donde no se haya reconocido que la información viaja mejor y más rápido por el "organigrama" informal, y que el conocimiento se reproduce y se enriquece no desde un grupo de gerentes que lo editan y controlan, sino desde los integrantes que lo producen y lo comparten.

David y Foray ${ }^{17}$ enfatizan que la característica crucial de la economía basada en el conocimiento está en el intercambio de información entre colegas. El desafío es lograr que Intranet opere como un fluidificador de la información para que ésta logre escapar de la comunidad de práctica que la produce y permear a todas las demás. Como dicen
Brown y Duguid, se trata de concebir la información a partir de su leakiness o stickiness, ${ }^{18}$ es decir, cómo fluye o cómo se "pega”.

Información, comunicación y conocimiento son tres conceptos diferentes pero están indisolublemente unidos; no es concebible la existencia de uno sin los otros, y para que los tres puedan recibirse, aplicarse, aprovecharse o trasladarse, el soporte facilitador es el contexto en el que se mueven los individuos y no la tecnología que apliquen. Dicen David y Foray que "el conocimiento en cualquier campo permite a quien lo posee tener la capacidad de actuar intelectual o físicamente. De esta forma, el conocimiento es esencialmente una cuestión de capacidad cognitiva. Por otro lado, la información consiste en datos estructurados que permanecen ociosos e inamovibles hasta que los utiliza alguien con el conocimiento suficiente para interpretarlos y procesarlos (...) El conocimiento está vinculado a individuos como tales y, por tanto, sólo será escuchado (...) o visto (...) por medio de la interac-
16:

Brown, J. y Duguid, P. 1998 "Organizing Knowledge".

Colifornia Management Review Vol. 40 , No 3

17:

David, P. y Foray, D. 2002

"Fundamentos Económicos de la Sociedad del Conocimiento". Revista Comercio Exterior, 56 (2). Pp. 476

Brown, J. y Duquid, op cit. 
Diana Marcela Blanco:: Es licenciada en Comunicación Social por la Pontificia Universidad Javeriana de Bogotá y está cursando el último año de la Maestría en Estudios Organizacionales en la Universidad de San Andrés en Buenos Aires.

Tiene experiencia como coordinadora del área de comunicaciones y marketing en empresas

de servicios en Colombia y Argentina.

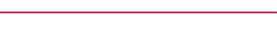

$$
\begin{array}{r}
19:: \\
\text { Ibid. Pp. 475. } \\
20: \text { : } \\
105 . \\
21: \text {.: } \\
\text { Brown y Duguid. Op. cit. Pp. } \\
\text { "Systems development will be } \\
\text { more like film production and } \\
\text { less like traditional engineering } \\
\text { (...) The comparison with } \\
\text { film production in the } \\
\text { organization of systems } \\
\text { development elucidates the } \\
\text { need for new skills, such as } \\
\text { telecommunication, artistic } \\
\text { and content skills, as well } \\
\text { as broad organizational } \\
\text { design and change- } \\
\text { management skills". Bansler, } \\
\text { J., Damsgaard, J., Scheepers, } \\
\text { R., Havn, E. y Thommesen, } \\
\text { J. 2000. "Corporate Intranet } \\
\text { Implementation: Managing } \\
\text { Emergent Technologies and } \\
\text { Organizational Practices". } \\
\text { Journal of the Association for } \\
\text { Information Systems, Vol. 1, } \\
\text { paper 10. } \\
22:: \\
\text { David, P. y Foray, D. Op. Cit. } \\
\text { Pág 474. }
\end{array}
$$

ción con quienes lo poseen. Plasmar (...) posibilita analizar y organizar el conocimiento de diferentes formas, así como aislar, clasificar y combinar distintos elementos". ${ }^{19}$

Es, por lo tanto, el individuo el que opera sobre los datos, el que puede tener la actitud de generar datos y compartirlos, pero son las organizaciones de las que forma parte las que pueden actuar como motivadoras o desmotivadoras de esa actitud. Aún en este mundo del free-rider y del teletrabajo, el conocimiento necesario para desarrollar una carrera laboral se genera dentro de la organización, ya sea trabajando dentro de ella o en otro tipo de relación de dependencia. Incluso la perspectiva actual de algunos autores -como Drucker, Brown y Duguid- es que las organizaciones se mantienen unidas con el objetivo de generar y renovar el conocimiento que se puede desarrollar en ellas, y no sólo por el acto de transacción. Drucker señala que el conocimiento pasa a ser un activo de las personas, pero Brown y Duguid sostienen que el conocimiento más relevante sigue siendo el que se produce a nivel de la organización y que, por lo tanto, no sería posible generar ninguna experiencia o conocimiento si no fuera por la colaboración de los miembros del equipo de trabajo.

Si las organizaciones no proporcionan la tecnología más adecuada para facilitar la generación y el intercambio de conocimiento, colocarán una traba fundamental para su desarrollo. Pero si no se ocupan de entender cómo viaja el conocimiento dentro de sus fronteras o, dicho de otra manera, cómo circula el conocimiento entre los nodos de su red, de poco les servirá la tecnología.

Organizar y hacer accesible y "usable" la información almacenada en la empresa es difícil porque el conocimiento, que se produce a partir de la experiencia de trabajar en grupos, como comunidades de práctica, difícilmente puede salir de allí. El conocimiento producido dentro del grupo que comparte códigos y un background específicos no es fácilmente comprendido por los que se encuentran fuera de esta comunidad y, muchas veces, es difícil encontrar la utilidad fuera del ámbito donde fue generado. Como señalan Brown y Duguid: "la facilidad o dificultad de mover el conocimiento es un reflejo del contexto social. Las tecnologías tienen un enorme papel, pero sólo si responden al contexto social. Para apoyar el flujo de conocimiento entre comunidades y organizaciones, el foco debe estar en acompañar a las comunidades y enriquecer su comunicación". ${ }^{20}$

Afortunadamente los trabajadores forman parte de varios y diversos equipos al realizar distintas tareas, lo que permite que la información se diversifıque un poco. Pero esta ventaja es difícil de reflejar en una herramienta como la Intranet, a pesar de que proporcione las aplicaciones que aparecen ideales para ello, como foros, blogs internos o videos. Algunos autores señalan que para que los trabajadores de hoy pudiesen hacer uso de estas aplicaciones tendrían que sumar a las capacidades requeridas para desarrollar su trabajo, cualquiera este sea, capacidades similares a las de un productor audiovisual. ${ }^{21}$ ¿Pero quiénes pueden realmente desarrollar con éxito esas capacidades?

Brown y Duguid sostienen, además, que es común asumir que el conocimiento explícito se mueve más fácilmente y que el conocimiento tácito se mueve con mayor dificultad. Otros autores señalan que para que se de transferencia de conocimiento tácito es necesario que exista confianza entre los integrantes del intercambio. No parece arriesgado afirmar que Intranet es una herramienta útil para la transferencia de conocimiento explícito: a través de ella es posible "la creación de objetos virtuales que pueden modificarse de manera indefinida y que son accesibles de inmediato para todos al mismo tiempo, facilita el trabajo y el aprendizaje colectivo".22 Entonces, el gran desafío para una Intranet es reproducir 
efectivamente el entorno informal de comunicación en el que habitualmente se da la transferencia de conocimiento tácito, ya que la confianza suele estar dada por la cercanía de las relaciones, los años de conocimiento y el contacto cara a cara, así como la certeza y la rapidez del feedback.

¿Pueden las aplicaciones de la Intranet replicar todo esto? Aparentemente la Intranet 2.0 podría tener las herramientas para lograrlo, siempre y cuando se utilice en un contexto de confianza organizacional y de valoración del conocimiento tácito. Pero la realidad es que muchos de los CEO de las corporaciones de hoy aún no entienden lo que es 2.0. ni están acostumbrados a que sus integrantes se expresen libremente. Por ello, muchas veces los directivos que solicitan y aprueban la Intranet y que esperan una alta participación y compromiso de los empleados son quienes imponen restricciones que impiden que el medio sea eficiente y no propician una adecuada cultura para ello.

\section{Para concluir}

Se tiende a asumir que el conocimiento viaja fácilmente; sin embargo, las organizaciones suelen están repletas de conocimiento paralizado, estacionado en una de las paradas del recorrido. El desafío está en movilizarlo y esto no se logra por la implantación de una herramienta, sino por la implantación de un nuevo modelo de organización: abierta, flexible, porosa, permeable, que permita, estimule y premie la peer-production sobre la producción controlada y editada de un grupo selecto. En una organización de este tipo Intranet en su versión 2.0 será un caso de éxito.

Como conclusiones generales, de los casos documentados estudiados podemos extraer que:

- Se reportan pocos casos de éxito en la implementación de Intranet (en su versión 2.0 especialmente).
- Se evidencia un gran vacío en la medición del impacto económico de la implementación de Intranet en la mayoría de las organizaciones.

- La creación de la Intranet es repartida en los diferentes departamentos de la empresa, aunque sobresale el área de sistemas $\mathrm{y}$ tecnología sobre las demás.

- Existe una tendencia a Intranets centralizadas en cuanto a su coordinación y a la dirección del proyecto de implementación (en contrapartida con Intranets por departamentos o por zonas territoriales).

- En las grandes organizaciones, privadas o públicas, se busca la participación del usuario a través de la generación de contenidos, la edición, el mantenimiento y la cogestión de la Intranet.

- Estas búsquedas y tendencias chocan con dificultades de desarrollo e implementación debido a que la organización no proporciona el contexto adecuado (comunicaciones cerradas al organigrama, miedo a la pérdida de control, cultura de lectura y subordinación en lugar de cultura de producción y empowerment).

"Los medios de comunicación organizacional deben ser mucho más que simples instrumentos para proveer contenidos si los directivos y comunicadores quieren que éstos tengan un impacto efectivo en el desarrollo de la empresa. Éstos deben estar alineados con la estrategia de comunicación y los fines organizacionales. La Intranet, por tanto, no puede ser una excepción, deberá contribuir, como todas las piezas comunicativas, al logro de las metas del negocio". ${ }^{23}$

Medir el impacto real de Intranet en la construcción y diseminación del conocimiento, se impone como desafío y como imperativo.::

Dafne Lavore::

Es licenciada en

Comunicación Social y

Organizacional por la

Universidad de Ciencias

Empresariales y Sociales

de Argentina y está

cursando el último

año de la Maestría en

Estudios Organizacionales en la Universidad de

San Andrés en Buenos

Aires. Ha trabajado

en el campo de la

comunicación empresaria, especialmente en el área de comunicación interna y en la organización de eventos.

\section{Ana Inés Pepe::}

Es licenciada en

Comunicación Social por la Universidad Católica del Uruguay y está cursando el último año de la Maestría en Estudios Organizacionales en la Universidad de San Andrés en Buenos Aires. Es consultora y co-directora de Contexto Comunicación Corporativa, y es docente de Comunicación Interna y de Gestión de la Imagen Corporativa en la Universidad Católica del Uruguay.

23::

Llano, A. 2006. "La Intranet en organizaciones Colombianas". Realidades Comunicativas. Volumen 9 No. 1. Pág. 107. 